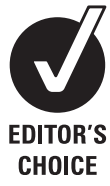

Singapore Eye Research Institute, Singapore National Eye Centre, Singapore, Singapore

${ }^{2}$ Department of Ophthalmology, Yong Loo Lin School of Medicine, National University of Singapore, Singapore,

Singapore

${ }^{3}$ Centre for Vision Research, University of Sydney, Sydney, Australia

${ }^{4}$ Centre for Eye Research Australia, University of Melbourne, Melbourne, Australia

\section{Correspondence to} Dr Anna C S Tan, Singapore National Eye Centre, 11 Third Hospital Avenue, Singapore 168751, Singapore: annacstan@gmail.com

Accepted 20 February 2012 Published Online First 9 March 2012

\title{
The impact of bilateral or unilateral cataract surgery on visual functioning: when does second eye cataract surgery benefit patients?
}

\author{
Anna C S Tan, ${ }^{1}$ Wan Ting Tay, ${ }^{1}$ Ying Feng Zheng, ${ }^{1,2}$ Ava Grace Tan, ${ }^{3}$ \\ Jie Jin Wang, ${ }^{3,4}$ Paul Mitchell, ${ }^{3}$ Tien Yin Wong, ${ }^{1,2,4}$ Ecosse Luc Lamoureux ${ }^{1,4}$
}

\begin{abstract}
Aim To examine the impact of bilateral or unilateral cataract surgery on visual functioning.

Methods The Singapore Malay Eye Study is a population-based study of 3280 Singapore Malay patients aged 40-80 years, of which 3225 had data available for inclusion. Cataracts were graded from digital lens photographs according to the Wisconsin scale. Study subjects were categorised as having: bilateral cataract surgery performed; unilateral cataract surgery performed with minimal cataract in the fellow eye; unilateral cataract surgery performed with significant cataract in the fellow eye; and bilateral cataract. Visual functioning was assessed using the modified VF-9 scale culturally adapted for Singaporean individuals, validated by Rasch analysis. The overall Rasch-modified vision-specific functioning score was compared across the four groups after adjusting for confounders such as age, gender, ocular and systemic comorbidities.
\end{abstract}

Results Persons with bilateral cataract had poorer visual functioning than those who had bilateral cataract surgery (mean visual functioning scores 3.38 vs 3.11, respectively, $p=0.029$ ). When compared with bilateral surgery, visual functioning improvements among patients with unilateral cataract surgery depended on the status of the fellow eye, with improvements only seen if the fellow eye had significant cataract (mean visual functioning scores 2.81 vs $3.25, p=0.019$ ) or poor visual acuity (mean visual functioning scores 2.78 vs $3.25, p=0.018$ ) after adjusting for confounders.

Conclusions Bilateral cataract surgery was associated with greater visual functioning over unilateral

cataract surgery when the fellow eye had a significant cataract or poor presenting visual acuity,

supporting the current practice of second eye surgery depending on the fellow eye's cataract status and visual acuity.

Cataract-induced visual impairment impacts negatively on quality of life, ${ }^{1-3}$ while cataract surgery has been shown to improve visual acuity, visual functioning and quality of life. ${ }^{4} 5$ Studies have shown that both bilateral and even unilateral surgery is both beneficial and cost effective. ${ }^{6-8}$

Most studies on cataract surgery outcomes have focused on visual acuity, although it is now clearly recognised that visual acuity improvement per se may not always lead to enhanced visual functioning and vision-dependant activities of daily living. Visual functioning is often influenced by patients' expectations and perceived outcomes, and is a better 'patient-centered' measure. Many scales have been developed to measure the patient's perceived visual functioning with respect to cataract and cataract surgery. ${ }^{9-12}$ Visual functioning and patients' perception of disability caused by cataract have been shown to be more important factors than surgeon preference or visual acuity in the final decision-making on whether cataract surgery should be performed.

The visual functioning (VF-14) scale is a popular scale that has been used to assess functional impairment in cataract patients. It is internationally validated, concise and easy to administer but has its limitations. ${ }^{13-15}$ Most previous studies, however, have used the mean or summary score of the VF-14 to generate an overall visual functioning score, ${ }^{16}$ and several studies have highlighted the limitations of that technique. ${ }^{14}$ 17-20 Rasch analysis is increasingly being considered as an alternative to traditional psychometric methods. The VF-14 and other shortened versions have been shown to be unidimensional, reliable and valid scales to assess vision-specific functioning in clinical and population-based samples. ${ }^{18} 20$

In this study, we examined the impact of unilateral and bilateral cataract surgery on visual functioning. In particular, we were interested to determine whether bilateral cataract surgery had greater benefit to visual functioning than unilateral cataract surgery, and if so, when was the best timing to have second eye surgery. Our data may be useful in determining resource allocation and public funding levels for cataract surgery.

\section{METHODS}

\section{Study population}

The Singapore Malay Eye Study (SiMES) is a population-based cross-sectional study of 3280 (78.7\% response rate) Malay individuals. The study population and procedures have been detailed elsewhere. $^{2} \quad 3 \quad 21-23$ An age-stratified random sampling procedure was used to select Malay people between the ages of 40 and 80 years living in the southwestern part of Singapore. Medical and socioeconomic data were recorded using a standardised questionnaire described previously. ${ }^{21}$ This study was conducted in accordance with the Declaration of Helsinki with ethics approval from the Singapore Eye Research Institute Institutional Review Board. 


\section{Vision assessment and definition of visual impairment}

Subjects underwent standardised examination procedures including visual acuity testing and a detailed clinical slit-lamp examination. The visual acuity of each eye was tested separately while subjects wore their habitual optical corrections (spectacles or contact lenses). Visual acuity was measured using a logarithm of the minimum angle of resolution (LogMAR) vision chart (Lighthouse International, New York, New York, USA) at a distance of $4 \mathrm{~m}$. If no letters could be read at $4 \mathrm{~m}$ the participant was moved closer to the chart; 3, 2 and $1 \mathrm{~m}$, consecutively. If no letters were identified on the chart, visual acuity was recorded as counting fingers, hand movemements, perception of light or no perception of light. Final refraction and best-corrected visual acuity was measured subjectively by a trained, certified study optometrist.

No or mild visual impairment was defined as best-corrected visual acuity of $6 / 18$ or greater. Moderate visual impairment was defined as visual acuity better than $6 / 18$ but worse than or equal to $6 / 60$. Severe visual impairment was defined as a visual acuity worse than 6/60. To assess bilateral visual impairment we divided our subjects into six categories: no or mild visual impairment in both eyes; no or mild visual impairment in one eye and moderate visual impairment in the fellow eye; no or mild visual impairment in one eye and severe visual impairment in the fellow eye; moderate visual impairment in both eyes; moderate visual impairment in one eye and severe visual impairment in the fellow eye; and severe visual impairment in both eyes.

\section{Definition of cataract surgery and cataract}

Relevant to our study the lens status and the presence of aphakia and pseudophakia were clinically assessed at the slitlamp examination. Cataract surgery was defined as the absence of the crystalline lens in at least one eye of a subject. ${ }^{23}$

After pupil dilation, lens photography was performed using a digital slit-lamp camera (Topcon model DC-1 with FD-21 flash attachment; Topcon, Japan) and a Scheimpflug retro-illumination camera (Nidek EAS-1000; Nidek, Japan). The slit-lamp photographs and the black and white retro-illumination photographs were used to assess the presence of cataract, according to the Wisconsin cataract grading system. The slitlamp and retro illumination photographs were graded by a single trained grader at the Centre for Vision Research, University of Sydney, with adjudication provided by a senior researcher and senior ophthalmologist for questionable cases. Adjudication was also provided for more difficult images with poor image quality, as well as confounding lesions or artifacts. Significant cataract was defined using the 'late definition' of the Wisconsin cataract grading system, which is nuclear opacity more than or equal to standard 4, cortical cataract more than or equal to $25 \%$ or posterior subcapsular cataract more than or equal to $5 \%$. Minimal cataract was defined as a nuclear grade less than 4, cortical cataract less than $25 \%$ or posterior subcapsular cataract less than $5 \%$.

\section{Visual functioning assessment}

The VF-11 is a modified form of the VF-14 validated to suit the local Singaporean cultural context. ${ }^{2} 18$ The visual functioning questions assessed the level of difficulty in performing activities of daily living. The numeric scale ranging from 0 (no difficulty) to 4 (unable to perform activity) was used to assess the difficulty of the following activities: seeing stairs, seeing street or shop signs, recognising people, watching television, cooking, playing cards (or mahjong), reading newspapers, completing lottery forms, reading small print, driving in the day, driving at night.

\section{Rasch analysis}

The modified VF-11 (VF-9) has previously been shown to be a unidimensional, reliable and valid scale to assess vision-specific functioning in this population using the Rasch model. ${ }^{2}{ }^{18}$ Rasch analysis is a modern psychometric method that mathematically describes the way respondents interact with items on a scale. In the Rasch model, the probability of a correct response is modelled as a logistic function of the difference between person ability (person measure) and item difficulty (item measure). Rasch analysis therefore provides a strict model that the structure of the responses should satisfy, rather than a simple statistical description of the responses. ${ }^{14} 1820$ A high person measure (in logits) indicates a high level of the assessed latent trait (eg, vision-specific functioning). Rasch analysis also provides insight into the psychometric properties of a scale, such as how well items fit the underlying latent trait being measured, how well items discriminate between the respondents, how well item difficulty targets person ability, and the appropriateness of the response scale used. ${ }^{14}$ Rasch analysis was performed using RUMM 2020 software (RUMM Laboratory, Perth, Australia). ${ }^{24}$ Winsteps software (V.3.61) ${ }^{25}$ was used to generate transformed individual person scores. ${ }^{17} 1921$

\section{Statistical analysis and comparison of visual functioning scores}

The characteristics of the study population, by cataract surgery and cataract status, were examined using proportions or means and $\mathrm{SD}$, and $\mathrm{p}$ values for differences between the groups were based on $\chi^{2}$ or $t$ test, respectively. The visual functioning scores after Rasch analysis in phakic subjects were compared with those who had unilateral and bilateral cataract surgery, after adjusting for confounding factors including age, gender, cardiovascular risk factors, current cigarette smoking status, low socioeconomic status and the presence of other eye diseases, including age-related macular degeneration, diabetic retinopathy or glaucoma. Using analysis of covariance, we obtained these crude and adjusted mean scores and associated $p$ values. The Rasch scores were then stratified according to the presenting visual acuity (in the better-seeing eye) to examine whether the score differences still existed. Visual acuity in operated eyes as well as the bilateral vision status of patients who had undergone cataract surgery was compared with subjects who were phakic Statistical analyses were performed using the statistical software SPSS V.13; $\mathrm{p}<0.05$ indicated statistical significance.

\section{RESULTS}

Of the original 3280 SiMES participants, 3225 were included in the analyses as they had the required socioeconomic, clinical and visual functioning data. Participants who were included tended to be younger compared with those who were excluded (58.6 \pm 11.0 vs $62.4 \pm 11.5$ years, $p=0.012$ ). Other than age, there were no significant differences in characteristics between these two groups.

The bilateral vision status as classified into the groups according to visual impairment is shown in table 1 . Of the subjects who had bilateral cataract surgery according to their presenting visual acuity, the majority, 98 (64.9\%), had no or mild visual impairment in both eyes and 34 (22.5\%) had no or mild visual impairment in one eye and moderate visual impairment in the fellow eye. Similarly, of the subjects who had unilateral cataract surgery with no cataract in the fellow eye, 18 (41.9\%) 
Table 1 Bilateral vision status of individual subjects within the group using presenting visual acuity $(\mathrm{p}<0.001)$

\begin{tabular}{|c|c|c|c|c|c|}
\hline \multirow[b]{2}{*}{ Bilateral vision status (\% of patients) } & \multirow[b]{2}{*}{$\begin{array}{l}\text { Bilateral cataract } \\
\text { surgery }\end{array}$} & \multicolumn{2}{|l|}{ Unilateral operated eye } & \multirow[b]{2}{*}{$\begin{array}{l}\text { Bilateral significant } \\
\text { cataract }\end{array}$} & \multirow[b]{2}{*}{$\begin{array}{l}\text { Bilateral minimal } \\
\text { cataract }\end{array}$} \\
\hline & & $\begin{array}{l}\text { With minimal } \\
\text { cataract in fellow eye }\end{array}$ & $\begin{array}{l}\text { With significant } \\
\text { cataract in fellow eye }\end{array}$ & & \\
\hline $\begin{array}{l}\text { No or mild visual impairment in both } \\
\text { eyes, } N=2267(70.3 \%)\end{array}$ & $98(64.9)$ & $18(41.9)$ & $18(20.7)$ & $191(37.1)$ & $1783(83.9)$ \\
\hline $\begin{array}{l}\text { No or mild visual impairment in one eye } \\
\text { and moderate visual impairment in the } \\
\text { other eye, } N=428(13.3 \%)\end{array}$ & $34(22.5)$ & $14(32.6)$ & $29(33.3)$ & $107(20.8)$ & $174(8.2)$ \\
\hline $\begin{array}{l}\text { No or mild visual impairment in one eye } \\
\text { and severe visual impairment in the other } \\
\text { eye, } N=78(2.4 \%)\end{array}$ & $2(1.3)$ & $7(16.2)$ & $15(17.2)$ & $18(3.5)$ & $21(1.0)$ \\
\hline $\begin{array}{l}\text { Moderate visual impairment in both eyes, } \\
N=310(9.6 \%)\end{array}$ & $10(6.6)$ & $1(2.3)$ & $8(9.2)$ & $124(24.1)$ & $124(5.8)$ \\
\hline $\begin{array}{l}\text { Moderate visual impairment in one eye and } \\
\text { severe visual impairment in the other eye, } \\
\mathrm{N}=96(3.0 \%)\end{array}$ & $6(4.0)$ & $1(2.3)$ & $13(15.0)$ & $47(9.1)$ & $19(0.9)$ \\
\hline $\begin{array}{l}\text { Severe visual impairment in both eyes, } \\
N=46(1.4 \%)\end{array}$ & $1(0.7)$ & $2(4.7)$ & $4(4.6)$ & $28(5.4)$ & $5(0.2)$ \\
\hline Total, $\mathrm{N}=3225$ & $151(4.7)$ & $43(1.3)$ & $87(2.7)$ & $515(16.0)$ & $2126(65.9)$ \\
\hline
\end{tabular}

had both eyes with no or mild visual impairment, while 14 $(32.6 \%)$ had no or mild visual impairment in one eye and moderate visual impairment in the fellow eye.

The differences in the demographics, ocular and systemic comorbidities are summarised in patients who have had cataract or have undergone unilateral or bilateral cataract surgery (tables 2 and 3). There was a significant difference in the Raschcalibrated visual functioning scores in those who had bilateral cataract surgery compared with those who had bilateral cataract (3.38 vs 3.11, $\mathrm{p}=0.029$ logits; table 4). However, after adjusting for confounders and stratifying the patients according to visual acuity, this difference was no longer statistically significant (3.25 vs 3.22 logits, $p=0.095)$. Comparing subjects with bilateral cataract surgery with those with minimal cataract, phakic subjects with minimal cataract had significantly higher visual functioning scores (3.25 vs 3.47 logits, $p=0.002$; table 3 ). However, with presenting visual acuity in the model, the differences were no longer significant.

Comparing bilateral with unilateral cataract surgery, the visual functioning scores were dependent on the status of the fellow eye. When the fellow eye had minimal cataract, the visual functioning scores were similar in subjects with unilateral and bilateral cataract surgery ( 3.25 vs 3.22 logits, $p=0.897$; table 5 ). Similarly, when the fellow eye had a presenting visual acuity that was better than 6/12 ( $\log M A R<0.3)$, there was also no difference in visual functioning scores when compared with subjects with bilateral cataract surgery (3.38 vs 3.25 logits, $\mathrm{p}=0.456$; table 5).

When the fellow eye had significant cataract, the visual functioning scores were statistically significantly poorer (2.81 vs 3.25 logits, $p=0.019$; table 5$)$. When the fellow eye had poor visual acuity, $(\leq 6 / 12$ or LogMAR $\geq 0.3$ ) the visual functioning scores were also statistically significantly poorer than in subjects who had bilateral cataract surgery ( 2.78 vs 3.25 logits, $p=0.018$; table 5).

\section{DISCUSSION}

Our key finding is that bilateral cataract surgery was associated with greater visual functioning over unilateral cataract surgery only when the fellow eye had a significant cataract or poor presenting visual acuity. Our findings support the current practice of second eye cataract surgery to be considered only when the fellow eye has significant cataract or reduced presenting visual acuity. As shown in our study and many others, cataract surgery results in improvements in visual acuity, visual functioning and reduces visual impairment. Our findings confirmed that visual acuity is the primary underlying mechanism underpinning vision-specific functioning, as shown previously. $^{7} 8$

Previous studies have demonstrated that consistent worsening unilateral and bilateral categories of visual impairment (ranging from mild to no visual impairment in both eyes to severe visual

Table 2 Comparisons of characteristics between subjects with bilateral cataract and those with bilateral surgery

\begin{tabular}{|c|c|c|c|c|c|}
\hline & $\begin{array}{l}\text { Bilateral cataract } \\
\text { surgery }(n=151)\end{array}$ & $\begin{array}{l}\text { Bilateral significant } \\
\text { cataract }(n=515)\end{array}$ & p Value $†$ & $\begin{array}{l}\text { Bilateral minimal } \\
\text { cataract }(n=2126)\end{array}$ & p Value $\dagger$ \\
\hline Age, years & $69.7(6.8)$ & $69.9(6.5)$ & 0.749 & $53.4(8.7)$ & $<0.001$ \\
\hline Gender, male & $77(51.0)$ & $227(44.1)$ & 0.134 & $1028(48.4)$ & 0.531 \\
\hline Age-related macular degeneration & $13(8.6)$ & $45(8.8)$ & 0.945 & $71(3.3)$ & 0.001 \\
\hline Diabetic retinopathy & $48(31.8)$ & $39(7.6)$ & $<0.001$ & $258(12.2)$ & $<0.001$ \\
\hline Glaucoma & $14(9.3)$ & $38(7.4)$ & 0.446 & $73(3.4)$ & $<0.001$ \\
\hline Any cardiovascular disease & $22(14.6)$ & $69(13.4)$ & 0.712 & $205(9.6)$ & 0.051 \\
\hline Current cigarette smoking & $13(8.6)$ & $85(16.5)$ & 0.016 & $493(23.2)$ & $<0.001$ \\
\hline Low socioeconomic status & $72(47.7)$ & $241(46.8)$ & 0.848 & $304(14.3)$ & $<0.001$ \\
\hline
\end{tabular}

Data are presented as mean (SD) or number (\%), as appropriate.

Cardiovascular disease is defined as a history of angina, myocardial infarction or stroke; low socioeconomic status is defined as having a primary or lower education and an individual monthly income less than $\$ S 2000$.

$t p$ Value to test for difference between bilateral cataract surgery compared with bilateral cataract and both eyes healthy, respectively, based on either a t test or $\chi^{2}$ test, as appropriate. 
Table 3 Comparisons of characteristics between subjects with bilateral and unilateral cataract surgery

\begin{tabular}{|c|c|c|c|c|c|}
\hline & \multicolumn{5}{|c|}{ Unilateral cataract surgery with } \\
\hline & $\begin{array}{l}\text { Bilateral cataract surgery } \\
(n=151)\end{array}$ & $\begin{array}{l}\text { Minimal cataract in } \\
\text { fellow eye }(n=43)\end{array}$ & p Value* & $\begin{array}{l}\text { Significant cataract in } \\
\text { fellow eye }(n=87)\end{array}$ & p Value* \\
\hline Age, years & $69.7(6.8)$ & $65.3(7.8)$ & $<0.001$ & $70.5(6.7)$ & 0.403 \\
\hline Age-related macular degeneration & $13(8.6)$ & $5(11.9)$ & 0.516 & $10(11.5)$ & 0.468 \\
\hline Diabetic retinopathy & $48(31.8)$ & $14(33.3)$ & 0.850 & $15(17.4)$ & 0.016 \\
\hline Glaucoma & $14(9.3)$ & $3(7.0)$ & 0.639 & $10(11.5)$ & 0.583 \\
\hline Low socioeconomic status & $72(47.7)$ & $16(37.2)$ & 0.224 & $36(41.4)$ & 0.347 \\
\hline Mean Rasch score (95\% CI) & 3.38 (3.17 to 3.58$)$ & 3.39 (3.02 to 3.77$)$ & 0.941 & $2.99(2.72$ to 3.25$)$ & 0.021 \\
\hline Adjusted mean Rasch score $(95 \% \mathrm{Cl}) \dagger$ & $3.25(2.99$ to 3.50$)$ & $3.22(2.78$ to 3.66$)$ & 0.897 & $2.81(2.46$ to 3.16$)$ & 0.019 \\
\hline
\end{tabular}

Data are presented as mean (SD) or number (\%), as appropriate.

Cardiovascular disease is defined as a history of angina, myocardial infarction or stroke; low socioeconomic status is defined as having a primary or lower education and an individual monthly income less than $\$ S 2000$.

${ }^{*}$ p Value to test for difference between bilateral cataract surgery (common) compared unilateral cataract surgery with a minimal cataract in fellow eye, significant cataract in fellow eye, presenting visual acuity worse than or equal to $6 / 12$ and presenting visual acuity better than $6 / 12$ in fellow eye, respectively, based on either a t test or $\chi^{2}$ test, as appropriate.

†Adjusted for age, gender, cardiovascular disease, current cigarette smoking status, low socioeconomic status and the presence of eye diseases (including age-related macular degeneration, diabetic retinopathy and glaucoma).

impairment in both eyes) was independently associated with poorer vision functioning. ${ }^{2}$ In our study the visual functioning scores in subjects with bilateral cataract surgery were better than in those with bilateral cataract, but this was not statistically significant once we adjusted for confounders. A possible explanation could be that the subjects, despite having a significant cataract as assessed clinically with the Wisconsin scale 'late' definition cut-off, $57.9 \%$ had no or mild visual impairment in at least one eye. This is an example in which cataract grading may not necessarily correlate with visual acuity and visual function.

However, visual functioning scores did correlate with bilateral visual impairment status. Subjects who had bilateral cataract surgery had better bilateral vision status, having no or mild visual impairment in either eye with lower rates of bilateral severe visual impairment than those with bilateral cataract.

When subjects who had bilateral cataract surgery were compared with those who had unilateral cataract surgery, the impact on visual functioning was associated with the status of the fellow eye. When the fellow eye had minimal cataract or good visual acuity $(>6 / 12)$, there was no difference in visual functioning scores. When the fellow eye had significant cataract or poor presenting visual acuity $(\leq 6 / 12)$, visual functioning was statistically significantly improved after bilateral cataract surgery. These findings support the benefits of second eye surgery in eyes with bilateral significant cataract, as demon- strated previously. ${ }^{7}$ This supports the guidelines in our centre and many others to use a visual acuity cut-off at approximately $6 / 12$ or worse as an indication for cataract surgery. On the other hand, second eye surgery may not result in improved visual functioning in patients with good visual acuity or minimal cataract in the fellow eye.

We found visual functioning to be poorer in those who had bilateral cataract surgery compared with those subjects who had not had surgery. The implications of cataract surgery, postsurgical recovery and other related co-existing ocular and systemic diseases may explain why subjects who had not undergone surgery had higher visual functioning scores than those who had bilateral cataract surgery.

The strengths of this study include its large sample, high response rate, and the use of standardised protocols previously shown to be applicable to the cultural context. ${ }^{2} 19$ The modified VF-9 scale that fits the Rasch model provides more accurate scoring of visual functioning compared with other studies that have used mean or summary visual functioning scores. ${ }^{22} 23$ Rasch analysis is increasingly considered the gold standard of modern scale validation, and can be used to look at the impact of cataract surgery on visual functioning. ${ }^{1}$ On the other hand, our study population included only Singaporean Malay individuals and our study findings cannot be generalised to other populations. Another limitation of our study is the lack of data on inter

Table 4 Comparisons of visual functioning and Rasch scores between subjects with bilateral cataract and those with bilateral surgery

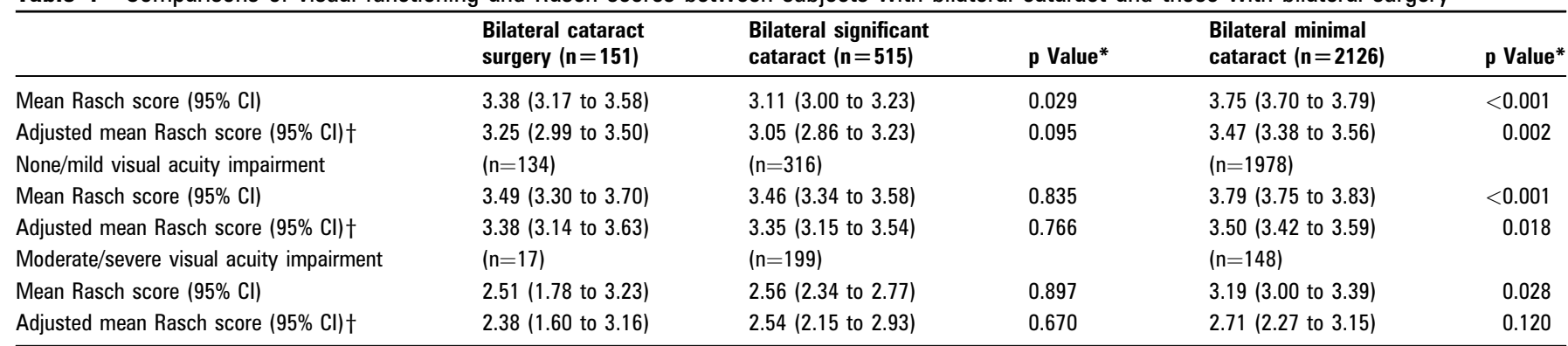

Data are presented as mean (SD) or number (\%), as appropriate.

Cardiovascular disease is defined as a history of angina, myocardial infarction or stroke; low socioeconomic status is defined as having a primary or lower education and an individual monthly income less than $\$ S 2000$.

*p Value to test for difference between bilateral cataract surgery compared with bilateral cataract and both eyes healthy, respectively, based on either a $t$ test or $\chi^{2}$ test, as appropriate.

†Adjusted for age, gender, cardiovascular disease, current cigarette smoking status, low socioeconomic status and the presence of eye diseases (including age-related macular degeneration, diabetic retinopathy and glaucoma). 
and intra-observer/interviewer reproducibility, but the Wisconsin cataract grading scale and VF-14 scale have previously been shown to be reliable, reproducible scales, and our observers and interviewers were masked as to the visual acuity of the patients. Our study is cross-sectional and visual functioning data before and after cataract surgery is therefore not available. We acknowledge this limitation and future work should consider a longitudinal design to record visual functioning scores before and after cataract surgery as a more robust assessment of postoperative visual functioning improvements. In addition, we did not collect data on the incidence of secondary cataract but we expect the rates in the general population to be low, thus not having a major impact on our results.

In summary, bilateral cataract surgery was associated with greater visual functioning over unilateral cataract surgery only when the fellow eye had a significant cataract or poor presenting visual acuity. Our findings suggest that second eye cataract surgery may not improve visual functioning if the fellow eye has minimal cataract or good visual acuity.

Contributors ACST researched data and wrote manuscript. WTT wrote the tables and performed statistical analysis of the data. YFZ researched the data. AGT graded the cataracts. JJW and PM performed statistical analysis, reviewed and edited the manuscript. TYW set up and supervised the project, contributed to discussion, reviewed/edited the manuscript. ELL performed statistical analysis, contributed to discussion, reviewed/edited the manuscript.

Funding This study was supported by the National Medical Research Council (0796/2003) and Biomedical Research Council (501/1/25-5). Additional support was provided by the Singapore Tissue Network, the Ministry of Health, Singapore. The sponsors had no role in the study design, acquisition of data, statistical analysis and interpretation, and the final presentation and publication of the study.

Competing interests None.

Ethics approval Ethics approval was provided by the Singapore Eye Research Institute Institutional Review Board.

Provenance and peer review Not commissioned; externally peer reviewed.

Data sharing statement Data are available on request from the corresponding author: annacstan@gmail.com.

\section{REFERENCES}

1. Finger RP, Fenwick $\mathrm{E}$, Marella $\mathrm{M}$, et al. The impact of vision impairment on visionspecific quality of life in Germany. Invest Ophthalmol Vis Sci 2011;52:3613-19.

2. Lamoureux EL, Chong E, Wang JJ, et al. Visual impairment, causes of vision loss, and falls: the Singapore Malay Eye Study. Invest Ophthalmol Vis Sci 2008;49:528-33.

3. Lamoureux EL, Chong EW, Thumboo J, et al. Vision impairment, ocular conditions, and vision-specific function: the Singapore Malay Eye Study. Ophthalmology 2008;115:1973-81

4. Varma R, Wu J, Chong K, et al. Impact of severity and bilaterality of visual impairment on health-related quality of life. Ophthalmology 2006;113:1846-53.

5. Yuen L, Do NH, Vu QL, et al. Cataract surgical outcomes, visual function and quality of life in four rural districts in Vietnam. Clin Experiment Ophthalmol 2011;39:119-25.

6. Busbee BG, Brown MM, Brown GC, et al. Cost-utility analysis of cataract surgery in the second eye. Ophthalmology 2003;110:2310-17.

7. Lundström M, Stenevi U, Thorburn W. Quality of life after first- and second-eye cataract surgery: five-year data collected by the Swedish National Cataract Register J Cataract Refract Surg 2001;27:1553-9.

8. Laidlaw DA, Harrad RA, Hopper CD, et al. Randomised trial of effectiveness of second eye cataract surgery. Lancet 1998;352:925-9.

9. Lundström M, Pesudovs K. Questionnaires for measuring cataract surgery outcomes. J Cataract Refract Surg 2011;37:945-59.

10. McAlinden C, Gothwal VK, Khadka J, et al. A head-to-head comparison of 16 cataract surgery outcome questionnaires. Ophthalmology 2011;118:2374-81.

11. Ronbeck M, Lundström M, Kugelberg M. Study of possible predictors associated with self-assessed visual function after cataract surgery. Ophthalmology 2011:118:1732-8.

12. Pesudovs K, Wright TA, Gothwal VK. Visual disability assessment: valid measurement of activity limitation and mobility in cataract patients. $\mathrm{Br} J$ Ophthalmol 2010;94:777-81.

13. Bilbao A, Quintana JM, Escobar A, et al. Responsiveness and clinically important differences for the VF-14 index, SF-36, and visual acuity in patients undergoing cataract surgery. Ophthalmology 2009;116:418-24 e1. 
14. Gothwal VK, Wright TA, Lamoureux EL, et al. Measuring outcomes of cataract surgery using the Visual Function Index-14. J Cataract Refract Surg 2010;36:1181-8.

15. Chiang PP, Fenwick E, Marella M, et al. Validation and reliability of the VF-14 questionnaire in a German population. Invest Ophthalmol Vis Sci 2011;52:8919-26.

16. Lau J, Michon JJ, Chan WS, et al. Visual acuity and quality of life outcomes in cataract surgery patients in Hong Kong. Br J Ophthalmol 2002;86:12-17.

17. Norquist JM, Fitzpatrick R, Dawson J, et al. Comparing alternative Rasch-based methods vs raw scores in measuring change in health. Med Care 2004;42(Suppl 1): $125-36$

18. Lamoureux EL, Pesudovs $\mathrm{K}$, Thumboo J, et al. An evaluation of the reliability and validity of the visual functioning questionnaire (VF-11) using Rasch analysis in an Asian population. Invest Ophthalmol Vis Sci 2009;50:2607-13.

19. Garamendi E, Pesudovs K, Stevens MJ, et al. The Refractive Status and Vision Profile: evaluation of psychometric properties and comparison of Rasch and summated Likert-scaling. Vision Res 2006:46:1375-83.
20. Gothwal VK, Wright TA, Lamoureux EL, et al. Rasch analysis of visual function and quality of life questionnaires. Optom Vis Sci 2009;86:1160-8.

21. Foong AW, Saw SM, Loo JL, et al. Rationale and methodology for a populationbased study of eye diseases in Malay people: the Singapore Malay Eye Study (SiMES). Ophthalmic Epidemiol 2007;14:25-35.

22. Lamoureux EL, Saw SM, Thumboo J, et al. The impact of corrected and uncorrected refractive error on visual functioning: the Singapore Malay Eye Study. Invest Ophthalmol Vis Sci 2009;50:2614-20.

23. Lavanya R, Wong TY, Aung T, et al. Prevalence of cataract surgery and post-surgical visual outcomes in an urban Asian population: the Singapore Malay Eye Study. Br J Ophthalmol 2009;93:299-304.

24. Andrich DLA, Sheridan B, Luo G, et al. RUMM 2020. Perth, Australia: RUMM Laboratory, 2003.

25. Linacre JA. User Guide to Winsteps: Rasch-model Computer Program. Chicago: Mesa Press, 2002 